2018

Transection and Crush Models of Nerve

Injury to Measure Repair and

Remyelination in Peripheral Nerve

\title{
Zhang, He
}

http://hdl.handle.net/10026.1/12276

10.1007/978-1-4939-7862-5_20

Springer New York

All content in PEARL is protected by copyright law. Author manuscripts are made available in accordance with publisher policies. Please cite only the published version using the details provided on the item record or document. In the absence of an open licence (e.g. Creative Commons), permissions for further reuse of content should be sought from the publisher or author. 
Transection and crush models of nerve injury to measure repair and remyelination in peripheral nerve

Xin-peng Dun and David B. Parkinson.

Plymouth University Peninsula Schools of Medicine and Dentistry, Plymouth, Devon, UK.

Address for correspondence:

Prof. David Parkinson, Plymouth University Peninsula Schools of Medicine and

Dentistry, John Bull building, Derriford, Plymouth, Devon, PL6 8BU, UK

E-mail: david.parkinson@plymouth.ac.uk

Running Head: Peripheral nerve transection and crush models. 


\section{i. Abstract.}

Injury to the peripheral nervous system begins a well characterised process within both neurons and Schwann cells to allow axonal regrowth, remyelination and functional repair. Models of peripheral nerve injury have been widely used to study the behaviour of Schwann cells, neurons and other cell types such as macrophages as the events of Wallerian degeneration and regeneration take place. The most commonly used approaches in rodent models to model nerve injury in human patients are sciatic nerve transection and nerve crush and both have well established time courses of demyelination, immune cell influx, axonal regrowth and remyelination. We describe the techniques of sciatic nerve surgery for transection and crush injury, together with methods for the analysis of events within peripheral nerve repair in these two models.

\section{ii. Key words.}

Peripheral nerve, sciatic, nerve transection, nerve crush, surgery, demyelination, remyelination, Schwann cell. 


\section{Introduction.}

While the peripheral nervous system (PNS) does possess the capacity to repair following injury, the degree of functional repair in human patients is very variable depending upon both the site and nature of the injury [1]. The need to model traumatic nerve injury in a rodent system has led to the adoption of two simple surgical approaches in the rat and mouse, nerve transection (also known as neurotmesis) and nerve crush (axonotmesis).

For complete nerve transection, the events of Wallerian degeneration distal to the site of injury proceed such as axonal loss, demyelination and immune cell influx, but the chances of a full functional repair are small. Following such an injury, the proximal and distal nerve ends typically retract away from each other and new tissue, the nerve bridge, forms between the two nerve stumps. Nerve transection injury has been widely studied to how the nerve bridge tissue is formed from nerve fibroblasts, Schwann cells and macrophages and how cell-cell interaction helps to guide axons in the correct trajectory across the bridge to re-enter the distal nerve stump [2-5]. The situation, however, following a crush injury is different as, while the events of Wallerian degeneration still occur distally, the structure of the nerve is maintained allowing axons to regrow through their original paths and re-innervate. Whereas measures of functional repair following sciatic nerve transection will most likely never show a full recovery, repair following a sciatic nerve crush close to the spinal cord will show full sensory and motor recovery at 21 days following injury in the mouse [4, $6]$.

Nerve transection and crush injury models have been widely used in mouse conditional null or over-expressing transgenic models and have identified key 
mechanisms in Schwann cells, neurons and macrophages that direct Wallerian degeneration, reprogramming of Schwann cells to a repair-competent phenotype, axonal pathfinding and remyelination of axons leading to functional repair [7-9]. In this Chapter, we describe the surgical methods involved in generating both sciatic nerve transection and crush injuries and show the analysis of such injuries from our and others work to illustrate their use. 


\section{Materials.}

\subsection{Equipment and reagents.}

1. Isoflurane anaesthetic set up for small animal surgery. The isoflurane vaporiser can either be wall mounted or as part of a compact anaesthesia system on a moveable trolley (see Note 1).

2. Sterile surgical tools: forceps (No. 5 size), scissors (fine and coarse), needle holder for sutures; a pair of delicate forceps for nerve crush (Fine Science Instruments; 0.4mm tip angled, Cat no. 11063-07)

3. Hair clipper for shaving skin area for surgery; preferably a re-chargeable unit (see Note 2).

4. Thermostatically heated surgical pad with rectal probe to measure core temperature of animal.

5. lodine sterilising solution for the skin (See Note 3.)

6. Sterile surgical drapes for both covering the animal and to place surgical instruments onto (see Note 4).

7. Sterile surgical gown, gloves and hat for person conducting surgery.

8. Surgical silk sutures (see Note 5).

9. Suitable local anaesthetic solution e.g. $0.025 \%$ bupivacaine (see Note 6).

10. Autoclip surgical staple applier and spare sterile clips (see Note 7).

11. Dry bead steriliser for sterilising instruments between each animal surgery (e.g. Steri 350)

12. Thermostatically controlled animal recovery chamber / heated pad (e.g. Thermacage advanced animal warming system). 


\section{Methods.}

\subsection{Sciatic nerve surgery.}

The protocol described below is as used in our laboratory and has been approved by the Plymouth University Animal Welfare and Ethical Review Board for work on the project licence of Prof. David Parkinson (see Note 8).

Sciatic nerve transection or crush surgery is carried out on animals, usually between the age of 2-3 months, and with a minimum weight of $20 \mathrm{~g}$. Surgical procedures are performed by two people, the surgeon and one person assisting to maintain sterility of the surgery. The assistant prepares the animal for surgery and removes the animal post-surgery; the surgeon performs the surgery on the prepared animal and only touches the sterile surgical instruments and the sterile surfaces of the surgical drapes. For best practice, the animal is anaesthetised and prepared for surgery (hair clipping and skin sterilisation) in a pre-op room, before being transferred to a separate room for surgery. Following surgery, the animal is transferred to a post-op room for recovery in the animal recovery chamber before transfer back to its cage.

1. All animals must be checked for general health before surgery and their weight before surgery recorded to check for any post-surgery weight loss. All animals undergoing surgery are moved to clean cages and labelled appropriately with animal number, sex, licence number and procedure to be performed.

2. The anaesthesia system and areas for surgery should be cleaned and free of any unnecessary clutter. The isoflurane vaporiser should be filled with isoflurane liquid using the key fill applicator. 
3. The heated animal recovery chamber and bead steriliser should be switched on to reach appropriate temperatures.

4. The individual performing the surgery should 'scrub up' using e.g. hybiscrub and put on a surgical hat, sterile surgical gown and gloves (See Note 9). The surgeon must not touch anything that is not sterile, if they do then they will need to change gloves.

5. The assistant will open packets containing the surgical drapes and the surgeon places the drapes on the benchtop. The heated surgical pad is placed on the drape by the assistant and switched on. The cloth drape may be pre-cut to fit around the surgical mask. The pack containing the surgical instruments is opened by the assistant and the surgeon places the sterile tools on the top of the sterile drape (see Fig. 1).

Steps 6-10 are performed by the surgical assistant:

6. The assistant switches on the oxygen supply and the flow rate is adjusted to 3 litres per minute (See Note 10). The anaesthetic gas is directed to the induction chamber and the isoflurane vaporiser set to $5 \%$. The first animal for surgery is placed into the induction chamber to be anaesthetised. Upon induction of anaesthesia, the breathing rate of the animal will reduce and the animal will lose its righting reflex. Reflexes (e.g. toe pinch to check for loss of reflexes) are used to ensure the animal is fully anaesthetised.

7. When the animal is anaesthetised, the assistant carefully removes the animal from the box and places the mouse onto a heated pad with its head in the anaesthetic mask. 
8. Turn the three-way valve to direct the anaesthetic toward the mask and reduce the oxygen flow to1.5 litres / $\mathrm{min}$ and the percentage of isoflurane to 2$3 \%$ to maintain the anaesthesia. Re-check level of anaesthesia of the animal by toe pinch to check reflexes.

9. The feet of the animal are gently secured by the use of clear tape and the clippers used to remove the fur in the area for surgery. Following hair clipping, use sticky tape to remove any loose fur from the area. The diluted iodine solution (see Note 3) is applied using a cotton bud to sterilise the area of skin where the incision will be made.

10. The assistant then places the anaesthetised animal onto the heated surgery pad (set to $37^{\circ} \mathrm{C}$ ) with the head of the animal inside the anaesthetic mask. The rectal thermoprobe is inserted to monitor animal core temperature.

The surgeon then performs steps 11-14

11. The surgeon places the sterile transparent drape onto the animal and makes the initial cut through the skin using scissors. The overlying gluteal and hamstring muscles are carefully separated to expose the sciatic nerve (see Fig. 1).

12. Following exposure of the sciatic nerve, the nerve may be transected or crushed. Typically, in the mouse, we perform nerve injury at $3 \mathrm{~mm}$ proximal to the trifurcation site of the nerve. For nerve transection experiments, the nerve is cut using fine scissors. For nerve crush surgery, a pair of round ended forceps is used to provide a reproducible crush injury to the nerve. The sciatic nerve is crushed once for 30 seconds and again for 30 seconds at the same site at right angle to the initial crush injury. In our hands, this approach 
ensures a complete crush of the nerve and degeneration of all distal axons of the nerve (See Fig. 1 and [10]).

13. The overlying muscle above the nerve is then closed using a suture. For our protocol (see Note 6), we then topically apply one or two drops of long lasting local anaesthetic onto the top of the sutured muscle $(0.025 \%$ bupivacaine $)$ for analgesia.

14. The skin is then closed using a stainless steel surgical clip using an Autoclip applier.

The assistant then performs Steps 15-17

15. The mouse is gently lifted from the heated surgery pad and transferred into the heated recovery chamber in the post-op area.

16. Once the mouse has fully regained consciousness (usually within 2-3 minutes after placing in the recovery chamber), it can be returned to a clean cage. Post-surgery, animals are given 'wet mash' to help with their feeding immediately post-surgery.

17. If performing surgery on more than one animal, then the next mouse is prepared for surgery as in Step 6. Surgical instruments are sterilised by the surgeon between surgeries using a dry bead steriliser.

18. Animals are monitored daily to check for general health, weight loss, any signs of infection around the surgery site and autotomy. If any animal exceeds the severity limit of the surgery protocol (e.g. weight loss or signs of autotomy), then the animal should be killed by an approved protocol (see Note 11). If animals are to be kept for longer periods post-surgery, then the surgical clips are removed at 10 days post-surgery once the skin has healed. 


\subsection{Analysis of functional recovery, axonal regrowth and remyelination.}

Both nerve transection and nerve crush injuries will begin the events of Wallerian degeneration distal to the site of injury. Sensory and motor axons will fragment and Schwann cells will begin to dedifferentiate and assume a repair-competent state. During this process, the blood-nerve barrier breaks down and immune cells enter the nerve and complete clearance of axonal debris and myelin.

Following sciatic nerve transection, even with re-joining of the nerve ends, there is very poor regeneration of axons into the distal stump of the nerve; consequently, measures of either motor or sensory functional recovery often show little improvement even after extended time-points. For sciatic nerve crush, however, as the structure of the nerve is maintained, Wallerian degeneration proceeds, axons regrow and are re-myelinated by Schwann cells. At 21 days post-crush injury in the mouse, functional testing shows a full sensory and motor recovery and from the animal's behaviour.

Functional testing of animals using either sensory or sensory/motor testing uses simple measures to test for recovery. In all cases, the person carrying out the test should be blinded to the genotype of the animal to prevent operator bias. Simple tests such as toe pinch (pressure), Hargreaves (temperature) or Von Frey (light touch) testing will monitor aspects of sensory recovery following injury. Another test such as toe spread will monitor motor function $[7,8]$.

Toe pinch test: This test measures sensory function by a local spinal reflex [11]. 
1. As the hind legs of the mouse are lifted from the ground by holding the tail, fine forceps are used to gently press the distal part of the individual toes (toes 3,4 and 5 for sciatic nerve injury).

2. Withdrawal of the leg from pressure applied to each toe is noted and represents functional recovery.

Hargreaves (Plantar) test: This is a widely used test to measure temperature sensitivity in mice and rats [12]. This tests the local spinal reflex of the peripheral nerves.

1. The mouse is placed on a platform that allows transmission of infrared heat.

2. The infrared heat source is placed under the platform (and $15-20 \mathrm{~cm}$ below the mouse), focused on one of the paws and switched on, allowing the heat source to send a beam of infrared radiation through the platform to reach the paw.

3. The mouse will move it paw as soon as it feels discomfort from the beam. The time it takes to do so is measured. The movement of the paw automatically switches off the heat source. Standard use of the test will use a cut off time (eg. 20 seconds) to prevent possibility of skin damage to the animal.

Von Frey test: This test measures mechanical nociception in mice and rats [9]. This test will measure loss of nociception following PNS injury and also allodynia (increased sensitivity) due to PNS dysfunction. Von Frey filaments are a set of nylon filaments of increasing diameter and thus buckling force to provide a range of forces for the measurement for mechanical nociception. 
1. Mice are placed on a wire mesh platform. The Von Frey filament is placed under the hindpaw. At the threshold the mouse will respond (paw flick/lick or vocalisation) and the filament is removed.

2. The test may be carried out several times on each mouse, spread over a period of several days to achieve a reliable average reading.

Toe spread test: the recovery of motor function in the injured sciatic nerve can be assessed using a simple toe spread test. Normally, when a mouse is lifted by the tail, the rear legs will extend laterally and the toes will spread in order to maximise the space between them. Loss of this reflex on the injured side of the animal and their recovery is used as a test for the return of motor function. [7, 11]

Another commonly used test to measure the recovery of sensory motor co-ordination is the sciatic functional index (SFI) or the modified version the static sciatic index (SSI) $[6,13,14]$. The SSI, due to its ease of use, has been widely used as a reliable method and uses a simple video camera assessment of toe spread and print length to generate a measure of recovery. Following surgery, the paw on the side of surgery shows a narrowing of the toe spread and increase in footprint length, both of which return to normal as recovery proceeds following crush injury. The uninjured contralateral side paw is used as an internal control measure. The procedure is carried out before surgery and is then performed 1 day following surgery and at 2-3 day intervals up to 21 days, at which point there should normally be a full return to normal SSI values.

As an example, loss of the Merlin protein in Schwann cells of the PNS leads to a complete lack of functional recovery [8]. Figure 2 shows an image from an SSI video 
at 21 days post-crush injury, showing a full recovery in a control mouse and no recovery in the Merlin null mouse.

1. The mouse is placed in a ventilated Perspex box $(30 \times 30 \times 30 \mathrm{~cm})$ and is filmed from underneath using a video camera. Filming takes no more than 2-3 minutes and the animal is then returned to its cage.

2. The video is viewed and, on a freeze frame, measurements made of the toe spread (toes 1-5) and print length on both the control side of the animal as reference and the side which has undergone surgery.

3. The SSI is calculated from the video measurements using the described formula [14]

As well as tests of functional recovery, analysis of the distal nerve at various timepoints can be used to assess Wallerian degeneration, axonal regrowth and remyelination. Assessment of the progression of axonal breakdown or regeneration can be made using cryostat sections or wholemount staining of whole distal nerve with a marker such as neurofilament [10]. To measure immune cell entry, cryosections of distal nerve may be immunolabelled with e.g. macrophage markers such as Iba1 or $\mathrm{F} 4 / 80[8,9,15,16]$.

For the preparation of cryostat sections:

1. The nerve is fixed overnight in $4 \%(w / v)$ paraformaldehyde in PBS pH 7.4.

2. The following day the nerve is washed 3 times (10 minutes each wash) in phosphate buffered saline (PBS) to remove any fixative and the sample dehydrated in $30 \%(\mathrm{w} / \mathrm{v})$ sucrose/PBS overnight.

3. The nerve is then embedded and frozen in Optimal Cutting Compound (OCT, 'Tissue Tek'). 
4. The tissue block is mounted on a cryostat and sections (usually 12-15 microns) are cut and placed onto positively charged (Superfrost) glass slides

5. Antibody staining is carried out as per manufacturer's instructions.

Most commonly, de-myelination and re-myelination of the distal nerve in injury models are assessed by using either semi-thin sections stained with toluidine blue or ultrathin sections used for transmission electron microscopy $[7-10,17]$. An example of an intact nerve together with a distal nerve at 14 days following nerve transection is shown in Figure 3.

\section{Notes.}

1. Isoflurane inhalation anaesthetic is preferable for this procedure as both induction of and recovery from anaesthesia is relatively rapid. Adjustments to the depth of anaesthesia can easily be made by increasing/decreasing isoflurane levels. Active scavenging of isoflurane should always be used, preferably with a built-in extraction or alternatively the use of an active scavenger unit in the anaesthetic circuit together with an activated charcoal cylinder (eg. Aldasorber) to remove the isoflurane. To ensure proper scavenging of isoflurane, the activated charcoal cylinder should be weighed before starting surgery and if the weight exceeds the manufacturer's limit (eg. $1400 \mathrm{~g}$ for Aldasorber), then a new cylinder installed. The isoflurane vaporiser should be serviced annually to be sure that the percentage isoflurane dispensed is correct. 
2. A cordless hair clipper (eg. Wella Contura) with a fine cutting head should be used. This minimises the likelihood of any cuts to the animal's skin during clipping.

3. A $1 / 10$ dilution of an iodine solution (eg. Vetasept, $7.5 \%$ povidone iodine) is used to sterilise the animal skin. Alcohol should not be used as this can cause temperature loss in the animal.

4. We routinely use a sterile cloth drape to cover the area of the surgery. For the drape to cover the animal during surgery, we use a sterile transparent plastic drape. This allows observation of the animal (eg. breathing rate) during surgery.

5. For suturing of muscle, we routinely use Ethicon M870 resorbable sutures size 8-0.

6. For post-operative analgesia, we use 1-2 drops of a bupivacaine solution, applied topically onto the surface of the sutured muscle before closing the skin with a surgical clip. This is prepared by making a $1 / 10$ dilution of a $0.25 \%$ bupivacaine hydrochloride solution (Marcaine). The choice of post-operative analgesia must be decided in consultation of both the local named veterinary surgeon and local regulations. A range of options for post-operative analgesia are used in labs doing this kind of procedure, including for example feeding mice pain reducing chow post-surgery, e.g. Bio Serv [16]).

7. For the autoclip surgical staple applier, for each surgery a new set of clips is inserted and the applier autoclaved with the other surgical instruments.

8. This protocol for sciatic nerve surgery has been passed by the Plymouth University Animal Welfare and Ethical Review Board, has been approved by the named veterinary surgeon and UK Home Office Inspector for the project 
licence held by David Parkinson under the UK Animals (Scientific Procedures) Act 1986.

9. We routinely use disposable sterilised surgical gowns for our work (Healthcare 365) to reduce the risk of post-surgical infection of the animal.

10. The higher oxygen flow rate (3-4 litres per minute) is used to induce anaesthesia rapidly in the induction chamber. Once the animal is anaesthetised and moved to the facemask for clipping then the flow rate can be reduced (1.5 litres / $\mathrm{min}$ ) for the maintenance of anaesthesia.

11. For this protocol, weight loss above $15 \%$ from the starting weight of the animal or autotomy following surgery resulting in damage to one nail bed of the animal represents the maximum severity level for the procedure. Any animals showing these effects will be killed by an approved method (e.g. cervical dislocation under the UK Schedule 1 procedure). 


\section{References.}

1. Zochodne, D.W., The challenges and beauty of peripheral nerve regrowth. J Peripher Nerv Syst, 2012. 17(1): p. 1-18.

2. Parrinello, S., et al., EphB signaling directs peripheral nerve regeneration through Sox2-dependent Schwann cell sorting. Cell, 2010. 143(1): p. 145-55.

3. Dun, X.P. and D.B. Parkinson, Visualizing peripheral nerve regeneration by whole mount staining. PLoS One, 2015. 10(3): p. e0119168.

4. Cattin, A.L., et al., Macrophage-Induced Blood Vessels Guide Schwann CellMediated Regeneration of Peripheral Nerves. Cell, 2015. 162(5): p. 1127-39.

5. Cattin, A.L. and A.C. Lloyd, The multicellular complexity of peripheral nerve regeneration. Curr Opin Neurobiol, 2016. 39: p. 38-46.

6. Yang, D.P., et al., Schwann cell proliferation during Wallerian degeneration is not necessary for regeneration and remyelination of the peripheral nerves: axon-dependent removal of newly generated Schwann cells by apoptosis. Mol Cell Neurosci, 2008. 38(1): p. 80-8.

7. Arthur-Farraj, P.J., et al., c-Jun Reprograms Schwann Cells of Injured Nerves to Generate a Repair Cell Essential for Regeneration. Neuron, 2012. 75(4): p. 633-47.

8. Mindos, T., et al., Merlin controls the repair capacity of Schwann cells after injury by regulating Hippo/YAP activity. J Cell Biol, 2017. 216(2): p. 495-510.

9. Roberts, S.L., et al., Sox2 expression in Schwann cells inhibits myelination in vivo and induces influx of macrophages to the nerve. Development, 2017. 144(17): p. 3114-3125.

10. Roberts, S.L., et al., The role of p38alpha in Schwann cells in regulating peripheral nerve myelination and repair. J Neurochem, 2016. 
11. Siconolfi, L.B. and N.W. Seeds, Mice lacking tPA, uPA, or plasminogen genes showed delayed functional recovery after sciatic nerve crush. J Neurosci, 2001. 21(12): p. 4348-55.

12. Hargreaves, K., et al., A new and sensitive method for measuring thermal nociception in cutaneous hyperalgesia. Pain, 1988. 32(1): p. 77-88.

13. Inserra, M.M., D.A. Bloch, and D.J. Terris, Functional indices for sciatic, peroneal, and posterior tibial nerve lesions in the mouse. Microsurgery, 1998. 18(2): p. 119-24.

14. Baptista, A.F., et al., A new approach to assess function after sciatic nerve lesion in the mouse - adaptation of the sciatic static index. J Neurosci Methods, 2007. 161(2): p. 259-64.

15. Napoli, I., et al., A central role for the ERK-signaling pathway in controlling Schwann cell plasticity and peripheral nerve regeneration in vivo. Neuron, 2012. 73(4): p. 729-42.

16. Mogha, A., et al., Gpr126/Adgrg6 Has Schwann Cell Autonomous and Nonautonomous Functions in Peripheral Nerve Injury and Repair. J Neurosci, 2016. 36(49): p. 12351-12367.

17. Quintes, S., et al., Zeb2 is essential for Schwann cell differentiation, myelination and nerve repair. Nat Neurosci, 2016. 19(8): p. 1050-9. 


\section{Figure legends}

Figure 1: A. Photograph of surgical set up for mouse peripheral nerve surgery. B-D close-up photographs of exposed mouse sciatic nerve (B), applying a nerve crush injury using forceps (C) and appearance of nerve post-crush (D).

Figure 2: Use of static sciatic index to measure functional recovery in mice following a sciatic nerve crush injury. A. Control wild-type mouse (NF2+/+) at 21 days postcrush injury. Side of surgery is marked by white arrow. Note normal appearance of foot as compared to contralateral control foot used as reference. B. In mice with a Schwann cell specific loss of the Merlin protein (NF2-/-), repair fails and the foot on the side of surgery shows no toe spread with a long print length. Such an appearance corresponds to a negative SSI measure.

Figure 3: Semithin sections of intact sciatic nerve $(A)$ and distal sciatic nerve at $14 d$ post-transection (B). Note complete breakdown of myelin in the transected nerve at this timepoint. Due to the nature of the injury (transection), there has been no axonal regeneration of remyelination of the distal nerve. Scale bar $75 \mu \mathrm{m}$. 